\title{
Attachment Patterns of Adolescent Patients with Mood Disorders and Their Parents
}

\author{
Bin $\mathrm{Hu}^{1}$ \\ ${ }^{1}$ West China School Of Basic Medical \& Forensic Medicine Sichuan University, Chengdu, 610041, China \\ 1274594517@qq.com
}

\begin{abstract}
During the growth of adolescents, adolescents are prone to mood disorders. The clinical symptoms of psychological disorders include recurrence, alternating or mixed emotions, and other related psychiatric symptoms. The most common is depressive episodes. Through the research and application of attachment theory, this paper will investigate the attachment mode of young people with psychological disorder, the attachment mode of parents, and the relationship with parenting mode, so as to better understand the pathological and psychological characteristics of young people with psychological disorders, and provide theoretical basis for clinical targeted psychotherapy. This article uses a cluster sampling method to select students from the first and third classes of the first high school in a certain city, and distribute the questionnaire of the Intimacy Experience Scale for their parents to complete and retrieve. The survey data was compared with survey data of outpatient and inpatient depression patients and their parents in mental health centers. The experimental research in this article shows that young people with emotional disorders have a negative attitude towards maintaining the emotional connection with others in the attachment relationship with their parents. They are worried about being abandoned and rejected, which is an insecure attachment.
\end{abstract}

Keywords: Depression, Attached Parent Pattern, Parenting, Pattern, Mood Disorder

\section{INTRODUCTION}

Mood disorder is also known as emotional disorder, which refers to a group of diseases characterized by major and continuous changes in feelings or mood caused by various reasons [1]. Almost all patients have a tendency to recurrent episodes, most of them can be relieved every time, and some patients have residual or chronic symptoms. Depression is a group of mental disorders. Patients usually have a low mood, loss of interest and pleasure, resulting in increased fatigue and decreased energy reduction in activities [2]. Most scholars believe that genetic factors account for a large proportion of the cause of mood disorders, and the induction of environmental factors and the influence of pathogenicity cannot even be ignored. Moreover, gender, age, personality traits, race, marriage, social, economic status, and education level, as well as life activities, stress, and physical factors are all considered risk factors for the onset of emotional disorders. A correct understanding of the risk factors that cause mood disorders will help to better prevent and determine the disease, and it will also help to complete a targeted treatment plan [3]. In addition, the formation of personality traits directly affects the family's intimate response to personal life attitudes and marital status. It is very important to study the relationship between the onset of psychological disorders and the family environment. In recent years, according to many related experimental studies, the family environment plays a very important role in the occurrence and onset of mood disorders. The overall family function of such patients is very weak, but the characteristics of the family environment are different, and the research on the influence of the etiology of psychological disorders is still rare [4].

Attachment research can be traced back to Freud's suggestion that initial interpersonal relationships will have a permanent impact on personal psychological development. Early childhood is an important period in a person's life. The experience of individuals, objects or objects obtained in the environment during early childhood will affect the lifestyle of adults to a certain extent. Freud also especially emphasized the importance of parent-child relationship in the experience of the development of personal character in the early years, and believes that parent-child relationship will become the prototype of various interpersonal relationship development in the future. However, Freud's hypothesis is based on the clinical observations of most psychiatric patients, and the specific measurement tools to verify this hypothesis are insufficient. Before the British psychiatrist Bowlby studied the children in the orphanage, he discovered that these children had lost their parents because of the war. Conceptual attachment refers to a particularly strong emotional connection 
between an infant and its caregiver [5-6]. Nostalgia has a direct meaning in maintaining the safety and survival of the baby, and its importance is no less than the control of diet and reproduction.

This article will investigate the nostalgia patterns of young people with mental disorders, the nostalgia patterns of parents, and the relationship with parenting styles to better understand the pathological and psychological characteristics of young people with mental disorders, and provide clinically targeted Psychotherapy provides theoretical basis. It is hoped that the experiments in this article can identify and prevent mood disorders as early as possible, and then formulate targeted treatment measures to provide a strong guarantee for the prognosis of patients.

\section{METHODS}

\subsection{Research Methods}

General survey questionnaire: a self-edited questionnaire for general population information such as children's gender, age, guardian's education, family monthly income, and marriage status. Youth Attachment Questionnaire: Revised by Liu Xi and Zhang Jianxin to include two subscales of family affection and attachment to friends. Each subscale is divided into four elements: affinity, negativity, dependence, and anxiety. There are 36 items in the questionnaire, which are scored on a scale of 1 to 4 . The reliability and validity of the questionnaire are good, with Cronbach's $\alpha$ coefficient of 0.70 and half reliability of 0.64 [7].

The Intimate Relationship Experience Scale was compiled by Brennan and others, and revised by Professor Li Tonggui of the Department of Psychology of Peking University, a Chinese version with an intimate experience scale. This can also be used to determine the close relationship between the subject and their parents, and to investigate the close relationship between parents and spouse. The reliability of avoiding retesting was 0.71 , and the reliability of retesting for uneasy maintenance was 0.72 . The questionnaire uses a 7-level scoring ring, "1" does not strongly agree, and "7" means strongly agree. This scale can be used to measure the attachment of young people after appropriate changes. In this study, in order to mainly evaluate the parent-child relationship, the original scale of "lovers" was replaced with "parents" [8].

\subsection{Preparation for Investigation}

Before the start of the study, all researchers received uniform standardized training. Before the questionnaire evaluation, the investigator explained the purpose and significance of the survey to each participant, and indicated the purpose and meaning of the survey on the first page of each page of the questionnaire, and actively assisted the participants in answering the questionnaire. After the researcher briefly explained all the questionnaires with simple instructions, the parents independently filled out the general questionnaire and the Intimacy Experience Scale, and the children filled out the adolescent attachment questionnaire independently. If the level of education is low and it is difficult to read and understand the topic, the researcher will explain and the respondent will fill in one by one. The emotional disorder group receives scale evaluation in the outpatient clinic, and the control group is adjusted by the teacher and filled out in the classroom [9-10].

\section{EXPERIMENT}

\subsection{Sample Data}

This article uses a group experiment divided into research groups and comparison groups. The research team used a convenient sampling method and selected outpatient and inpatient adolescent depression patients and their parents in the mental health center. Participation criteria:

- The diagnosis is confirmed by a registered doctor who is a professional or above;

- Patients should be 14-19 years old,

- Education of middle school or above, and have certain Chinese reading ability.

Exclusion criteria: device-induced brain disease, syndrome, bipolar disorder and depression. A total of 36 cases were registered, including 19 men and 17 women. In the control group, the students in the third class of the first high school in a certain city and their parents were regarded as the normal control group. Include benchmarks: no mental illness, brain qualitative illness, and family experience of mental illness. Finally, 33 cases including 23 teenagers and 10 girls were registered. Regarding age, gender and education level, there were no statistically significant differences between the study group and the comparison group.

\subsection{Research Design}

This study first analyzed the literature on adolescent mood disorders and parental attachment patterns, clarified the research ideas, and clarified the research content. Laid a foundation for the development of this research and the writing of thesis. The questionnaire survey method is also called the form-filling method, that is, the questionnaire is prepared and then distributed to the subjects of the research survey through a paper method. In this study, a total of 70 questionnaires were distributed and 70 valid questionnaires were recovered. Researchers conduct data analysis and word processing based on the answers to the questionnaire. Although the questionnaire can learn a lot of information, there are also situations where the information is incomplete or not specific and in-depth. Therefore, on the basis of the questionnaire survey, the researchers also used the literature research method, the purpose is to more comprehensively and accurately understand the 
relationship between adolescent mood disorder and parental attachment patterns and some opinions. The analysis of the following data will organically unify the results of the questionnaire and the literature research, and analyze the relationship between the current adolescent mood disorder and parental attachment patterns.

\section{DISCUSSION}

\subsection{Comparison of Differences between the Study Group and the Control Group}

From the perspective of avoidance of attachment and attachment anxiety, the scores of adolescent depression patients are higher than those of the control group, and the statistical gap between the two is obvious. Compared with the normal control group, the mothers of the research group had higher scores in the state of attachment anxiety, and the statistical difference between the two was more significant. However, as shown in Table 1, there was no statistically significant difference in attachment avoidance and attachment anxiety between the fathers of the research group and the comparison group. It can be seen from Table 1 that the friend dependence coefficient of children with emotional disorders has a negative relationship with the dimension of parental attachment anxiety. According to research, disturbed parents tend to adopt parenting methods such as negation, excessive control, and lack of warmth. These inadequate parenting methods always make people feel inferior to others, low independence, and it is difficult to establish good relationships with friends. The degree of dependence is low, and it is easy to fall into panic when leaving parents, causing negative feelings.

Table 1. Comparison of differences between the study group and the control group

\begin{tabular}{|l|c|c|c|c|}
\hline \multicolumn{1}{|c|}{ project } & $\begin{array}{c}\text { Depression group } \\
(\mathrm{n}=36)\end{array}$ & Control group(n=33) & $\mathrm{t}$ & $\mathrm{p}$ \\
\hline Child attachment avoidance & $73.19 \pm 16.632$ & $62.76 \pm 13.899$ & 2.814 & 0.006 \\
\hline Child attachment anxiety & $66.25 \pm 12.914$ & $51.97 \pm 13.692$ & 4.477 & $<0.001$ \\
\hline Mother attachment anxiety & $72.06 \pm 14.228$ & $57.42 \pm 12.811$ & 4.392 & $<0.001$ \\
\hline
\end{tabular}

\subsection{Regression Analysis of Experimental Results}

Father's parenting method: The first level is to avoid the father's attachment to the child as an independent variable, and the avoidance child's attachment to the child is used as a dependent variable for regression analysis. The second level is to use the father's avoidance as an independent variable, and the third level is In the case of regression analysis using father's warmth of affection as a dependent variable, the third level is to avoid father and use affective warmth as an independent variable, and children's attachment as a dependent variable in forced regression analysis. The results showed that the father's parenting style did not play an intermediate role in avoiding the father's attachment to the child.

Mothers' parenting methods: regression analysis shows that after adopting the emotional warming breeding method, the explanatory rate of the regression model for children's attachment avoidance increased from $23.8 \%$ to $36.7 \%$, and the regression coefficients are all meaningful $(-0.91, \mathrm{P}<0.01),(-0.11, \mathrm{P}<0.0) 5)$ is not enough $(-0.286, \mathrm{P}>0.96)$, which plays a role of complete mediation. For mothers who refuse to deny parenting laws, the regression coefficients of models are very important $(0.356, \mathrm{P}<0.05 ; 0.337, \mathrm{P}<0.05)$. The way of refusal to raise children shows that the mother's avoidance has an impact on the child's avoidance. The intermediary role is reached, as shown in Table 2.

Table 2. Regression analysis of parental rearing style

\begin{tabular}{|c|c|c|c|c|c|c|c|c|c|}
\hline \multirow{2}{*}{ project } & \multicolumn{3}{|c|}{1} & \multicolumn{3}{c|}{2} & \multicolumn{3}{c|}{3} \\
\cline { 2 - 9 } & Beta & $\mathrm{t}$ & $\mathrm{p}$ & Beta & $\mathrm{t}$ & $\mathrm{p}$ & Beta & $\mathrm{t}$ & $\mathrm{p}$ \\
\hline $\begin{array}{c}\text { level } \\
\text { one }\end{array}$ & 0.534 & 3.158 & 0.004 & 0.488 & 3.114 & 0.004 & 0.488 & 3.114 & 0.004 \\
\hline
\end{tabular}




\begin{tabular}{|c|c|c|c|c|c|c|c|c|c|}
\hline $\begin{array}{c}\text { level } \\
\text { two }\end{array}$ & -0.527 & -3.099 & 0.005 & -0.491 & -3.135 & 0.004 & 0.392 & 2.375 & 0.024 \\
\hline $\begin{array}{c}\text { level } \\
\text { three }\end{array}$ & 0.447 & 2.234 & 0.035 & 0.286 & 1.717 & 0.96 & 0.356 & 2.197 & 0.036 \\
\cline { 2 - 10 } & -0.165 & -0.822 & 0.419 & -0.411 & -2.467 & 0.02 & 0.337 & 2.085 & 0.046 \\
\hline
\end{tabular}

This paper also investigated the influence of parents of experimental class students on adolescent mood disorder,the results are shown in Figure 1. From the perspective of parents' subjective wishes, it is believed that the parental attachment pattern has a great influence on adolescent mood disorder. $64.42 \%$ of parents believe that parental attachment patterns have a greater impact on adolescent mood disorders, $20.28 \%$ of parents believe that parental attachment patterns have a decisive influence on adolescent mood disorders, only $1.13 \%$ of parents think that the impact is not significant, and only $0.24 \%$ of parents It is believed that the parental attachment pattern has no effect on mood disorders in adolescence.

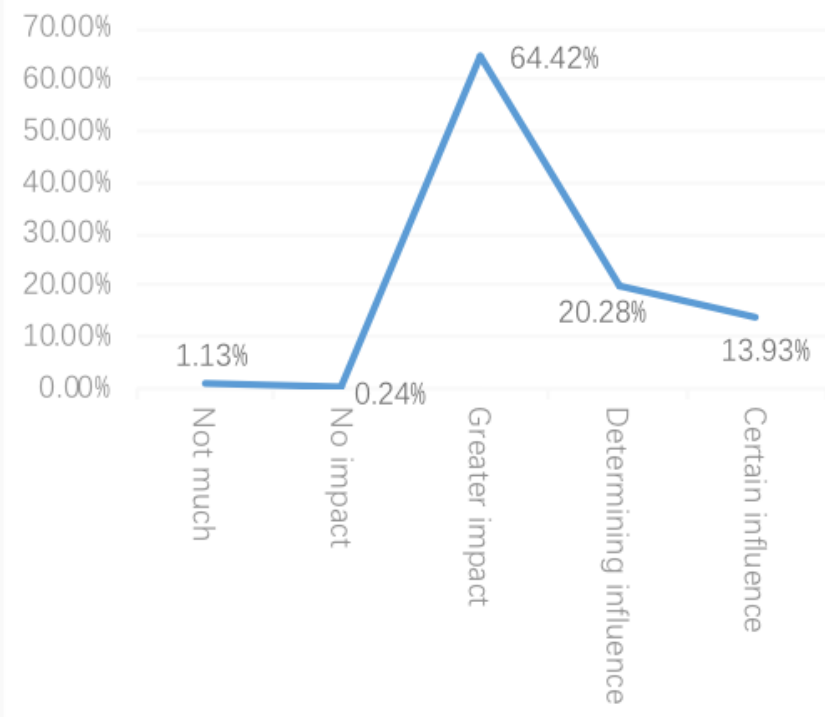

Figure 1. The influence of parental attachment patterns on adolescent mood disorders

\section{CONCLUSIONS}

Mood disorders are often referred to as affective disorders, also known as emotional psychosis. The clinical features are mainly abnormal changes in mood and feelings, which in most cases are accompanied by actions, cognition, communication with others, and abnormal moods. Psychophysiology and other obstacles and changes are mainly manifested in several extreme moods of mania and depression. The main symptoms of the patient's mood disorder are a series of signs and symptoms for several weeks or months, which seriously interfere with the patient's life and social functions, and the recurrence pattern is easy to be cyclic or periodic. All, in the current medical field, psychological disorders are not so-called isolated diseases, but are classified as a syndrome, and their causes and causes are almost the same. According to the research and investigation of this article, the characteristics of children's mood disorder are love for the family, low affinity, high negativity, and low affinity for friends' attachment, and the friend dependence coefficient of children with emotional disorders is related to the father's attachment degree. Therefore, attention should be paid to family therapy for children with heart disorders. Parents should control their feelings, show sufficient tolerance and tolerance, create a good family atmosphere, and promote the establishment of a safe attachment model for children.

\section{REFERENCES}

[1] Fenglian Z, Junling Y, Ranji C. Effect of Hypoxic Injury in Mood Disorder[J]. Neural Plasticity,2017, (2017-6-22), 2017, 2017:1-10.

[2] Elisabeth C, Adkins D E, Crowell S E, et al. An epigenetic pathway approach to investigating associations between prenatal exposure to maternal mood disorder and newborn neurobehavior[J]. Development and Psychopathology, 2018, 30(03):881-890.

[3] Parker G, Bassett D, Outhred T, et al. Defining melancholia: A core mood disorder[J]. Bipolar Disorders, 2017, 19(3):235.

[4] Mason B, Koh A, Van Enkevort E, et al. Gut Microbiota Distributions Predict Mood Disorder Symptoms and Mediate Dietary Interactions[J]. Biological Psychiatry, 2017, 81(10): S273-S274. 
[5] Jha K K, Chaudhary D P, Rijal T, et al. Delayed Stevens-Johnson Syndrome Secondary to the Use of Lamotrigine in Bipolar Mood Disorder[J]. Indian Journal of Psychological Medicine, 2017, 39(2):209-212.

[6] Zhang L, Shu M, An B, et al. Biparental incubation pattern of the Black-necked Crane on an alpine plateau[J]. Journal of Ornithology, 2017, 158(3):697-705.

[7] Spitoni G F, Zingaretti P, Giovanardi G, et al. Disorganized Attachment pattern affects the perception of Affective Touch[J]. entific Reports, 2020, 10(1):9658.

[8] Şaban Karayağız, Timuçin Aktan, Lider Zeynep Karayağız. Parental Attachment Patterns in Mothers of Children with Anxiety Disorder[J]. Children, 2020, 7(5):46.

[9] Okumura A, Ida S, Ito S, et al. Parental Awareness of Young Children's Pattern of Ionic Beverage Consumption[J]. Pediatrics International, 2018, 60(10):969-973.

[10] Lee A, Rattigan L, Gallagher D, et al. GP218 Growth patterns in a paediatric outpatient clinic and its association with child eating behaviours and parental feeding style[J]. Archives of Disease in Childhood, 2019, 104(Suppl 3): A119-. 